\title{
LncRNA ANRIL promotes multiple myeloma progression and bortezomib resistance by EZH2-mediated epigenetically silencing of PTEN
}

\author{
Li-Hui YANG, Peng DU, Wei LIU, Li-Kun AN, Jian LI, Wen-Yi ZHU, Shuo YUAN, Lei WANG, Lei ZANG* \\ Department of Orthopedics, Beijing Chao-Yang Hospital, Beijing, China \\ *Correspondence: zanglei19879@163.com
}

Received February 5, 2021 / Accepted March 30, 2021

\begin{abstract}
Multiple myeloma (MM) is a plasma cell malignancy of bone marrow. In the present study, we aimed to study the function and potential mechanism of the antisense non-coding RNA in the INK4 Locus (ANRIL) in MM. The expression levels of ANRIL in MM patients and healthy donors were evaluated by quantitative real-time polymerase chain reaction (qRT-PCR). The effects and mechanisms of ANRIL in MM were evaluated by cell viability assay, BrdU incorporation assay, tumor xenograft model, flow cytometry, western blot, RNA immunoprecipitation (RIP), transcriptome RNA sequencing, and chromatin immunoprecipitation (ChIP). We found that ANRIL was upregulated in MM patients and cell lines, and associated with advanced international staging system (ISS) stage and poor overall survival. Enforced ANRIL expression promoted proliferation and tumor xenograft growth of MM cells, while knockdown of ANRIL exhibited opposite effects. Moreover, ANRIL overexpression increased the half-maximal inhibitory concentration (IC50) of bortezomib and reduced bortezomib-induced apoptosis in MM cells. ANRIL was found to accumulate in the nuclei of MM cells, and interact with EZH2 by RIP assay. Transcriptome RNA sequencing identified PTEN as a target of ANRIL in MM cells. In the ChIP assay, knockdown of ANRIL reduced EZH2 occupancy and H3K27me3 binding to the promoter region of PTEN. Furthermore, EZH2 knockout or PTEN restoration abrogated the effects caused by ANRIL overexpression in MM cells. Our results indicated that ANRIL exerted oncogenic functions and conferred chemoresistance of MM cells by EZH2-mediated epigenetically silencing of PTEN.
\end{abstract}

Key words: ANRIL, multiple myeloma, bortezomib, PTEN

Multiple myeloma (MM) is the second most common hematologic malignancy arising from the plasma cells of bone marrow. It takes up $1 \%$ of all cancers and 10\% of all hematological malignancies [1]. The age-adjusted incidence rate for $\mathrm{MM}$ is about 4/100,000 [2]. The average age of MM patients at the time of diagnosis is $>65$, with less than $2 \%$ of patients younger than 40 [3]. Diagnosis of MM requires evidence for either $10 \%$ or more clonal plasma cells in bone marrow or a biopsy proved plasmacytoma and the existence of one or more myeloma-related end-organ damages [4]. Survival of MM patients reached a great improvement over the past decades, which increases from less than 3 years to 5-7 years now [5]. However, MM is a highly heterogeneous disease, and the survival of MM patients largely depends on host factors such as tumor burden and response to therapy. For example, a small subgroup of MM patients (approximately 15\%) exhibit really poor outcomes (median survival $<2$ years) [6]. The initial treatments for MM patients consist of high-dose therapy of bortezomib, lenalidomide, and dexamethasone (VRD) following autologous stem cell transplantation (ASCT) if eligible. Despite these aggressive treatments, almost all MM patients will eventually relapse. Thus, there is an emerging needing to understand the molecular mechanism of MM tumorigenesis and find new targets for MM treatment.

Long non-coding RNAs (lncRNAs) are a wide class of RNA transcripts longer than 200 nucleotides in length but without any protein-coding potential. There are increasing proofs that lncRNAs are involved in the tumorigenesis, metastasis, and chemoresistance of cancers [7].

For example, cytosolic lncRNA P53RRA induces cell-cycle arrest, apoptosis, and ferroptosis by interacting with G3BP1 and regulating p53 [8]. In lung cancer, LINC00336 regulated by ELAVL1 inhibits ferroptosis by sponging miR-6852 and regulating CBS [9]. Dysregulation of lncRNAs is found in MM patients $[10,11]$. Moreover, a variety of lncRNAs are reported to enroll in the initiation and progression of MM. For example, MALAT1 is upregulated in MM patients and 
forced MALAT1 expression promotes proliferation, migration, and survival of MM cells [12]. ST3GAL6-AS1 is high expressed in MM patients compared with normal plasma cells [13]. Silencing ST3GAL6-AS1 reduces cell proliferation and induces apoptosis of MM cells by regulating the MAPK pathway. Furthermore, dysregulation of lncRNAs is a potential prognostic biomarker for MM patients. For example, Zhou et al. found a four-lncRNA-based prognostic signature to divide MM patients into high- and low-risk groups [10]. In the present study, we are aimed to identify lncRNAs that play a role in the tumorigenesis of MM.

The antisense non-coding RNA in the INK4 Locus (ANRIL, also known as CDKN2B-AS1) is a $3.8 \mathrm{~kb}$ long non-coding RNA located at 9p21.3 of the human genome. ANRIL attracts much attention because it lies at the opposite direction of three important tumor suppressor genes ( $\mathrm{p} 19^{\mathrm{ARF}}, \mathrm{p} 15^{\mathrm{INK} 4 \mathrm{~b}}$, and $\left.\mathrm{p} 16^{\mathrm{INK} 4 \mathrm{~A}}\right)$ at the INK4b-ARFINK4a cluster. These three tumor suppressors are vital for cellular functions and frequently dysregulated in cancer cells [14]. Though the INK4b-ARF-INK4a cluster is deleted or silenced in nearly $40 \%$ of human cancers, a variety of studies demonstrate that ANRIL has pro-oncogenic properties [15]. For example, ANRIL is found to overexpress in acute myeloid leukemia (AML) patients [16]. Knockdown of ANRIL leads to a reduction of glucose uptake and suppression of AML cell maintenance by regulating the AdipoR1/AMPK/SIRT1 pathway. In T-cell acute lymphoblastic leukemia (T-ALL), ANRIL is apparently upregulated in T-ALL samples and regulates cell proliferation, migration, and invasion of T-ALL cells by sponging miR-7-5p and increasing TCF4 expression [17]. Notably, ANRIL is reported to upregulate in MM patients, and polymorphism of ANRIL is connected with tumor relapse of MM patients after autologous stem cell transplant, however, the exact role of ANRIL is to be elucidated [18, 19].

In the present study, we evaluated the possible role of ANRIL in MM and potential underlying molecular mechanisms. We found that ANRIL was elevated in MM patients and cell lines, and associated with advanced ISS stage and poor overall survival. Enforced ANRIL expression promoted proliferation and tumor xenograft growth of MM cells, while ANRIL knockdown exhibited opposite effects. Furthermore, ANRIL activated Akt signaling by EZH2-mediated epigenetically silencing PTEN. Silencing EZH2 or restoring PTEN expression abolished the effects caused by ANRIL overexpression. Our results indicated that ANRIL exerted oncogenic functions and promoted chemoresistance of MM cells. ANRIL might be a novel prognostic marker and therapeutic target for MM.

\section{Patients and methods}

Patient samples. Written informed consents were obtained from all patients or healthy donors who participated in the study. The study protocol involving human subjects was approved by the ethics committee of the Beijing Chao-Yang Hospital (SYSK (Jing) 2018-0012). Bone marrow aspirate samples from 80 newly diagnosed MM patients and 20 healthy donors were collected from March 2014 to May 2016 in Beijing Chao-Yang Hospital. These samples were fresh frozen by liquid nitrogen and maintained at $-80^{\circ} \mathrm{C}$ for use. Follow-up continued for 5 years after the patients were diagnosed with MM.

Cell culture. MM cell lines RPMI-8226, U266, MM.1S, and NCI-H929 were purchased from the American Type Culture Collection (ATCC). Human MM cell line MOLP-8 was obtained from DSMZ (Braunschweig, Germany). Human MM cell lines KMS-20, KMS-11, and KMS-27 were recently obtained from the JCRB Cell Bank (Osaka, Japan). Cells were cultured in RPMI-1640 medium containing 10\% fetal bovine serum (Hyclone, USA), $100 \mathrm{U} / \mathrm{ml}$ penicillin, and $100 \mu \mathrm{g} / \mathrm{ml}$ streptomycin. Cells were maintained in a humid atmosphere supplemented with $1 \% \mathrm{CO}_{2}$ at $37^{\circ} \mathrm{C}$. Subculture of cells was realized by adding fresh medium or centrifuging and reseeding.

Plasmid constructs, lentivirus packaging, and infection. Forced expression of ANRIL was achieved by cloning the full length of ANRIL into the pCDH lentivirus vector (System Biosciences \#CD510B-1). Empty pCDH vector was used as EV control. Short hairpin RNAs (shRNAs) specifically targeting ANRIL (sh-ANRIL-1 and sh-ANRIL-2) were inserted into the pLKO.1 lentiviral vector and a non-targeting sequence was cloned into the pLKO.1 vector as sh-NC control. Short guide RNAs targeting EZH2 (sgEZH2-1 and sgEZH2-2) were constructed by introducing into the lentiCRISPRv2 vector (Addgene, plasmid \#52961). A non-targeting sequence was cloned into the lentiCRISPRv2 vector as sgNC control. For lentivirus packaging, lentiviral plasmids were introduced into HEK293T cells with helper plasmids by Lipofectamine 3000 (Invitrogen, USA). The virus was harvested at 24, 48 , and $72 \mathrm{~h}$ post-transfection and stored at $-80^{\circ} \mathrm{C}$ for use. Transient transfection was realized according to the protocol of Lipofectamine 3000 (Invitrogen, USA). DNA sequences for shRNAs and sgRNAs were displayed as below: sh-ANRIL-1, 5'-TCGAAAGTCTTCCATTCTTCAAA-3', sh-ANRIL-2, 5'-ATGAAAAAGGAAGAAAGGAAAGC-3', sgEZH2-1, 5'-CATCATCATTATATTGACCA-3', sgEZH2-2, 5'-TTTACATAACATTCCTTATA-3'.

Quantitative real-time polymerase chain reaction (qRT-PCR). Total RNA was extracted by TRIzol reagent (Invitrogen, USA). Exosomal RNA was extracted by miRNeasy Micro kit (Qiagen, USA). The first strand of cDNA was synthesized by HiScript 1st Strand cDNA Synthesis Kit (Thermofisher, USA). RT-PCR was conducted according to the protocol of SYBR Premix Ex Taq kit (Takara, Japan) on a Real-time PCR Detection System (CFX96, Bio-Rad). The expression of ANRIL was normalized to GAPDH. The primer sequences for ANRIL were: forward, 5'-AGCAGAAGGTGGGCAGCAGAT-3'; reverse, 5'-GCAGGCAGGTAGTCCAGTGGTT-3' 
Cell viability assay. CellTiter-Glo Viability Assay kit (Promega \#G7572) was used to measure cell viability. The cells and CellTiter-Glo reagents were placed at room temperature for $30 \mathrm{~min}$. Next, cells and CellTiter-Glo reagents were mixed together at a ratio of 1:1. Then, cells were lysed on an orbital shaker for $5 \mathrm{~min}$ and placed at dark for $15 \mathrm{~min}$ avoiding light. The luminescence signal at $490 \mathrm{~nm}$ was recorded on a microplate reader. All samples were repeated in triplicates.

BrdU incorporation assay. Cells were incubated with $20 \mu \mathrm{M}$ BrdU for $3 \mathrm{~h}$ at $37^{\circ} \mathrm{C}$. Then cells were washed with PBS once and incubated with BrdU antibody (Cell Signaling \#5292, 1:1000) for $1 \mathrm{~h}$ at room temperature. After washing the cells with PBS once, incubation with Alexa Fluor 488 mouse IgG (Cell signaling \#4408, 1:1000) followed for $1 \mathrm{~h}$ at room temperature avoiding light. DAPI was used to stained the cells for $10 \mathrm{~min}$ at room temperature. The BrdU incorporated cells were viewed under the fluorescence microscope.

Tumor xenograft model. The protocol of animal studies was reviewed and approved by the ethics committee of the Beijing Chao-Yang Hospital (SYSK (Jing) 2018-0012). RPMI-8226 cells $\left(2 \times 10^{6}\right)$ transduced with ANRIL or EV control, or U266 cells $\left(2 \times 10^{6}\right)$ transduced with sh-ANRIL-1, sh-ANRIL-2, or sh-NC were subcutaneously implanted into 8 -week-old nude mice. Tumor xenografts were allowed to grow for 4 weeks, and tumor volume was measured every four days. The mice were sacrificed at last, then tumor xenografts were dissected out and weighed. Tumor volume was calculated according to the formula: $\left(\right.$ length $\times$ width $\left.^{2}\right) / 2$.

Flow cytometry. Cells were dispersed as single cells, then $1 \times 10^{6}$ cells were incubated with Annexin V-FITC at $4^{\circ} \mathrm{C}$ for $30 \mathrm{~min}$, and DAPI at $4^{\circ} \mathrm{C}$ for $15 \mathrm{~min}$ in a dark. The fluorescence at $488 / 530 \mathrm{~nm}$ was measured using flow cytometry (BD Biosciences).

Western blot. Protein lysates were extracted from tissue samples and cultured cells by RIPA buffer (Beyotime, China) containing protease inhibitors (Roche, USA). Protein concentration was measured using a BCA kit (Thermo Fisher, USA). Protein was separated by $10 \%$ SDS-PAGE, then transferred to nitrocellulose membranes. The membranes were blocked with 5\% non-fat milk for $1 \mathrm{~h}$ at room temperature, then stained with primary antibody at $4{ }^{\circ} \mathrm{C}$ overnight and secondary antibody for $1 \mathrm{~h}$ at room temperature. The specific primary antibodies were listed below: Cleaved Caspase-3 (Cell Signaling \#9664, 1:1000), GAPDH (Cell Signaling \#5174, 1:1000), p-Akt (Ser473) (Cell signaling \#9271, 1:1000), Akt (Cell Signaling \#9272, 1:1000), PTEN (Cell Signaling \#9188, 1:1000), EZH2 (Cell Signaling \#5246, 1:1000). The second antibody was anti-rabbit IgG HRP (Cell Signaling \#7074; 1:4000).

RNA immunoprecipitation (RIP). RIP was conducted according to the protocol of the Magna RIP RNA-Binding Protein Immunoprecipitation Kit (Millipore, USA). In brief, cell extracts were incubated with beads for $6 \mathrm{~h}$, then washed with RIP wash buffer 5 times. Then the beads were stained with EZH2 antibody (Cell Signaling \#5246, 1:50) or IgG (Cell
Signaling \#3900, $1: 50$ ) at $4^{\circ} \mathrm{C}$ overnight. Then RNA pulled down was evaluated by qRT-PCR.

Transcriptome RNA sequencing. Total RNA from RPMI-8226 cells transducing with ANRIL and EV was extracted using TriPure (Roche, USA). The cDNA library was synthesized and sequenced at Beijing Novel Bioinformatics (https://en.novogene.com/) following the Illumina standard protocol. Fastp, Fastqc (v0.11.5), and HTSeq V0.6.1 were used to analyze data. The differences between the two groups were evaluated by DESeq $\mathrm{R}$ package (1.10.1). A p-value $<0.05$ and $\mid \log _{2}$ Fold Change $\mid \geq 2$ were used to select genes different expressed.

Chromatin immunoprecipitation (ChIP). ChIP assay was performed using the EZ-ChIP kit (Millipore, USA). In brief, DNA-protein crosslinks were produced by treating cells with formaldehyde for $10 \mathrm{~min}$. Then cells were sonicated to DNA fragments and incubated with EZH2 antibody (Cell Signaling \#5246, 1:50) or H3K27me3 (Cell Signaling \#9733, 1:50). The precipitated DNA was evaluated by qRT-PCR.

Statistical analysis. GraphPad Prism 8.0 was used to analyze data. A two-tailed Student's t-test was used to evaluate the difference between the two groups. One-way ANOVA was used to compare the difference between multiple groups followed by Bonferroni's post hoc test. The half-maximal inhibitory concentration (IC50) of bortezomib was calculated using the nonlinear regression model of GraphPad Prism 8.0. A p-value $\leq 0.05$ was considered statistically significant.

\section{Results}

LncRNA ANRIL was upregulated in MM patients and predicted poor prognosis. To disclose the potential function of ANRIL in MM, we first evaluated the expression of ANRIL in MM patients. The levels of ANRIL in 80 newly diagnosed MM samples and plasma cells of 20 healthy controls were measured by qRT-PCR. We found that ANRIL was significantly upregulated in MM patients compared with healthy controls (Figure 1A). Furthermore, the MM patients were divided into different groups according to the international staging system (ISS). We found that ANRIL expression was apparently increased in MM patients with advanced ISS stage (Figure 1B). Besides, the association between ANRIL and prognosis of MM patients was evaluated by KaplanMeier analysis. High expression of ANRIL was closely correlated with shorter overall survival (Figure 1C). The expression of ANRIL in MM cell lines was further evaluated by qRT-PCR. Compared with plasma cells from healthy control, ANRIL was dramatically overexpressed in U266, MM.1S, and KMS-20 cells (Figure 1D). Taken together, our results indicated that ANRIL was upregulated in MM patients and cell lines, and predicted a poor prognosis.

Enforced ANRIL expression promoted proliferation and tumor xenograft growth MM cells. The possible functions of ANRIL in MM were tested by overexpression of ANRIL in two MM cell lines (RPMI-8226 and NCI-H929), which 

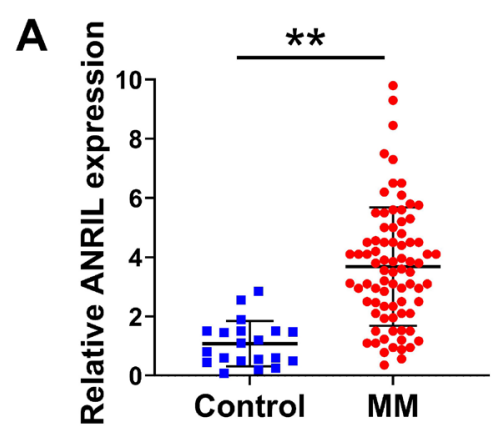

\section{C}

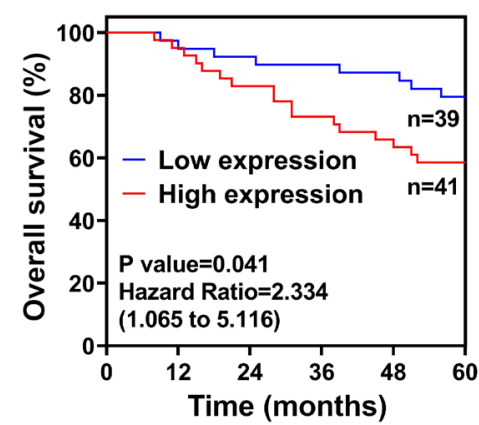

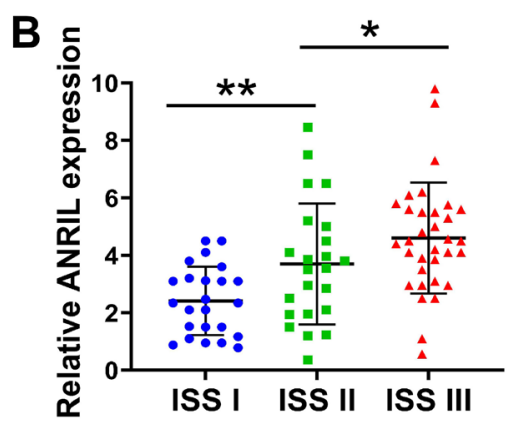

$\mathrm{D}$

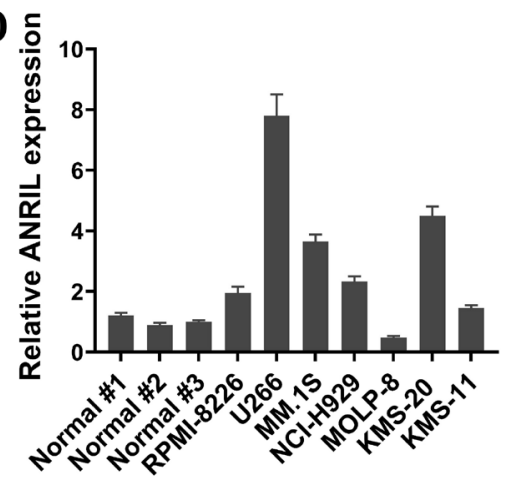

Figure 1. LncRNA ANRIL is upregulated in MM patients and predicted poor prognosis. A) relative expression of ANRIL in bone marrow aspirate samples from $80 \mathrm{MM}$ patients and 20 healthy donors was evaluated by qRT-PCR. B) Relative expression of ANRIL in MM patients according to the international staging system (ISS) stage. C) Kaplan-Meier analysis of overall survival in MM patients according to ANRIL expression. D) Relative expression of ANRIL in MM cell lines and normal plasma cells was evaluated by $\mathrm{qRT}-\mathrm{PCR} .{ }^{*} \mathrm{p} \leq 0.05,{ }^{* *} \mathrm{p}<0.001$

showed low endogenous ANRIL expression. The full length of ANRIL was cloned into a lentiviral expression vector, then introduced into RPMI-8226 and NCI-H929 cells. We could observe that ANRIL was obviously overexpressed in MM cell lines (Figure 2A). To evaluate the influence of ANRIL on cell proliferation of MM cells, cell viability assay and BrdU incorporation assay were conducted. Compared with empty vector (EV)-transduced cells, ANRIL overexpressed RPMI-8226 and NCI-H929 cells exhibited an apparently increased cell viability at day 6 (Figure 2B). In the BrdU incorporation assay, the overexpression of ANRIL in RPMI-8226 and NCI-H929 cells facilitated BrdU incorporation compared with EV control (Figures 2C, 2D). These results indicated that ANRIL overexpression promoted the proliferation of MM cells in vitro. In order to verify this in vivo, RPMI-8226 cells transduced with ANRIL expression lentiviral or EV control were injected into nude mice and assayed for tumor xenograft growth. We found that enforced ANRIL expression dramatically promoted the growth of RPMI-8226 xenografts in nude mice (Figure 2E). After implantation for 4 weeks, tumor xenografts of ANRIL overexpressing RPMI-8226 cells showed larger tumor volume and increased tumor weight (Figures 2F, 2G). Taken together, our results suggested that enforced ANRIL expression promoted proliferation and tumor xenograft growth of MM cells.
Knockdown of ANRIL suppressed proliferation and tumor xenograft growth of MM cells. ANRIL was found to overexpress in MM patients and cell lines in our study (Figures 1A, 1D). To elucidate the influence of ANRIL overexpression in MM, we also knocked down ANRIL in a MM cell line (U266) which showed high endogenous expression of ANRIL. Two short hairpin RNAs (shRNAs) specifically targeting ANRIL (sh-ANRIL-1 and sh-ANRIL-2) were introduced into U266 cells by lentiviral infection. A non-targeting control (sh-NC) was used as a control. Our results indicated that ANRIL expression was successfully downregulated by these two shRNAs, though to a different extent (Figure $3 \mathrm{~A}$ ). In cell viability assay, we found that knockdown of ANRIL by sh-ANRIL-1 or sh-ANRIL-2 evidently reduced the viability of U266 cells at day 4 and 6 compared with sh-NC (Figure 3B). In the BrdU incorporation assay, knockdown of ANRIL in U266 cells reduced BrdU incorporation (Figures 3C, 3D). These results indicated that knockdown of ANRIL suppressed the proliferation of MM cells. In the tumor xenograft model, U266 cells transduced with sh-ANRIL-1 or sh-ANRIL-2 showed decreased tumor growth, volume, and weight (Figures 3E-3G). Collectively, our results demonstrated that knockdown of ANRIL suppressed proliferation and tumor xenograft growth of MM cells. 
A

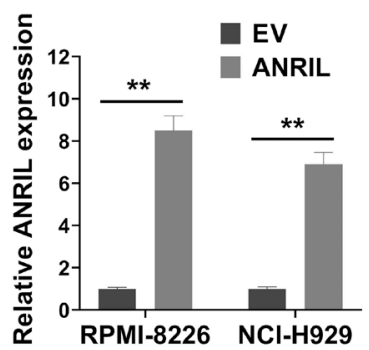

B

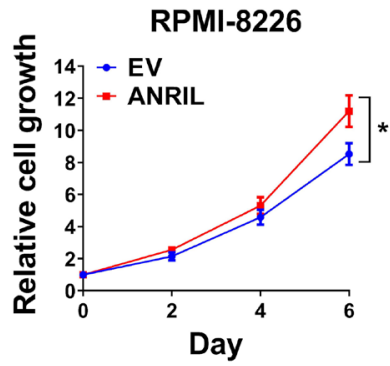

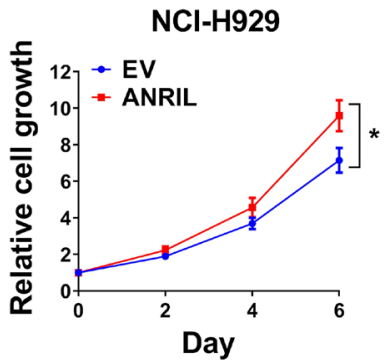

C
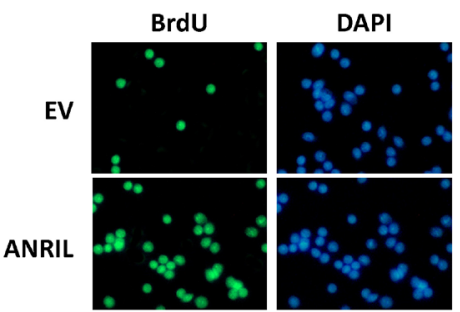

RPMI-8226

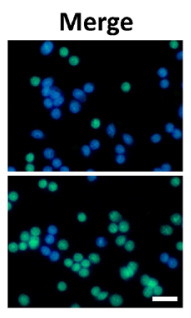

E

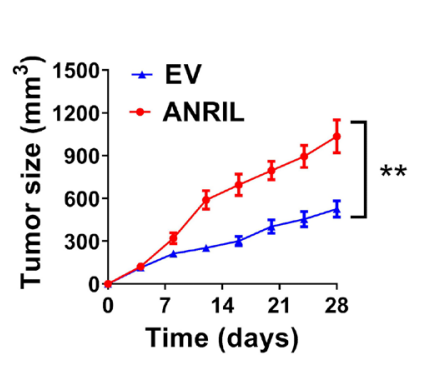

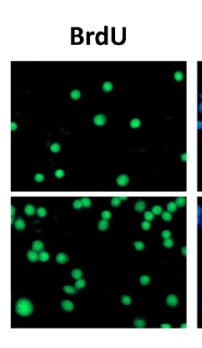

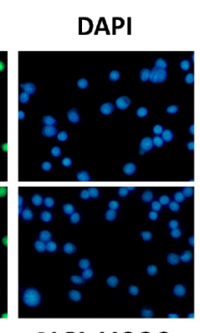

$\mathrm{NCl}-\mathrm{H} 929$
EV

ANRIL

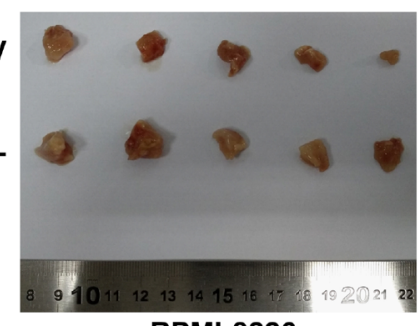

G

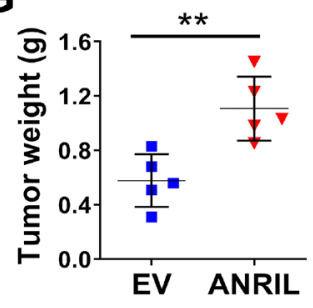

Figure 2. Enforced ANRIL expression promotes proliferation and tumor xenograft growth of MM cells. A) RPMI-8226 and NCI-H929 cells were introduced with ANRIL or EV control, then the relative expression of ANRIL was evaluated by qRT-PCR. B) RPMI-8226 and NCI-H929 cells (2,500/ well) transduced with ANRIL or EV control were seeded in 96-well plates, then cell viability was evaluated at day 0, 2, 4, and 6. C, D) RPMI-8226 and NCI-H929 cells $\left(5 \times 10^{5} /\right.$ well $)$ transduced with ANRIL or EV control were seeded in 6-well plates for the BrdU incorporation assay. Represent images (C) and percentage of BrdU positive cells $(\mathrm{D})$ are shown. Scale bar $=50 \mu \mathrm{m} . \mathrm{E}-\mathrm{G}$, RPMI-8226 cells $\left(2 \times 10^{6}\right)$ transduced with ANRIL or EV control were implanted into nude mice for 4 weeks. Tumor growth $(E)$, volume $(F)$, or weight $(G)$ are shown. ${ }^{\star} p \leq 0.05,{ }^{\star *} p<0.001$

ANRIL overexpression increases bortezomib tolerance of MM cells. Bortezomib (BTM) is an FDA-approved drug used for treating newly diagnosed MM. Resistance to bortezomib-based therapy is frequently observed in MM patients [20]. To explore if ANRIL was involved in the resistance of bortezomib, we overexpressed ANRIL in MM cells and measured the IC50 of bortezomib by cell viability assay. Our results indicated that ANRIL overexpression evidently increased bortezomib tolerance of RPMI-8226 and NCI-H929 cells (Figure 4A). The IC50 of bortezomib increased approximately twofold after cells were introduced with ANRIL. In addition, RPMI-8226 and NCI-H929 cells were treated with 10 or $20 \mathrm{nM}$ bortezomib for flow cytometry. We found that ANRIL overexpression obviously reduced the percentages of Annexin-V and PI double-positive subsets in RPMI-8226 and NCI-H929 cells treated with bortezomib, suggesting a decrease in apoptosis (Figures 4B, $4 \mathrm{C}$ ). In western blot analysis, the levels of apoptosis marker cleaved caspase- 3 were evaluated. Our results indicated that RPMI-8226 and NCI-H929 cells introduced with ANRIL showed lower levels of cleaved caspase-3 when treated with bortezomib (Figure 4D). Above all, our results suggested that ANRIL increased bortezomib tolerance of MM cells, and this might due to the suppressing of cell apoptosis.

ANRIL epigenetically silences PTEN by interacting with EZH2. To elucidate the underlying molecular mechanism of ANRIL in MM, we detected the subcellular location of ANRIL in RPMI-8226 and NCI-H929 cells by qRT-PCR. We found that ANRIL was mainly existed in the nuclei of RPMI-8226 and NCI-H929 cells, indicating that ANRIL 
A

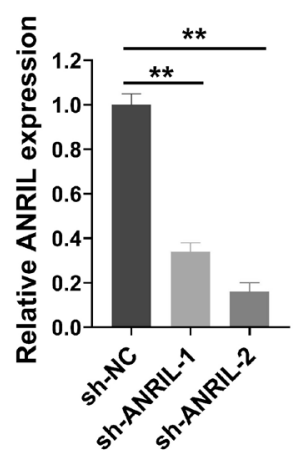

D

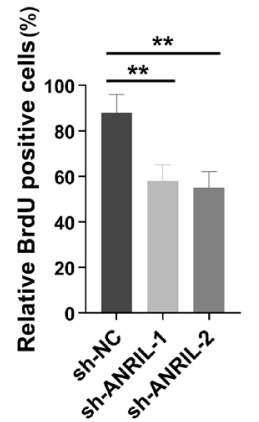

B

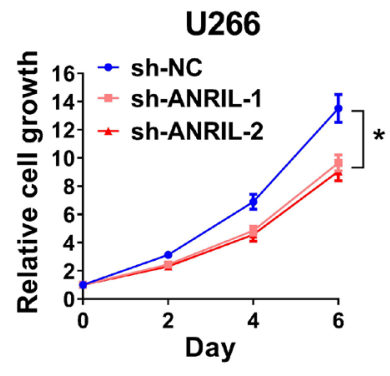

E

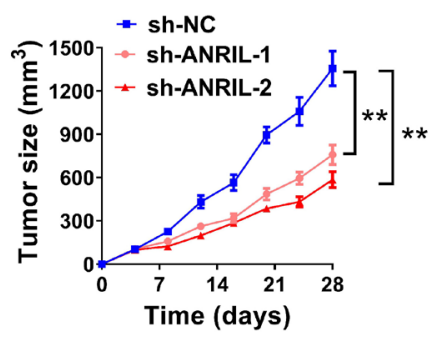

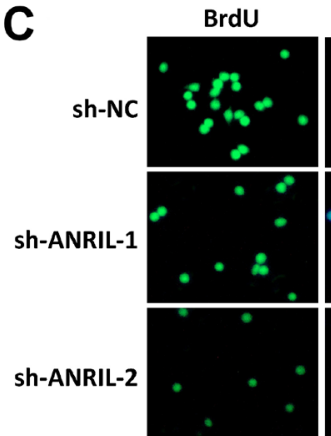

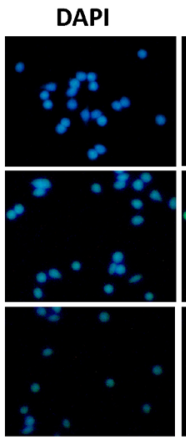

F

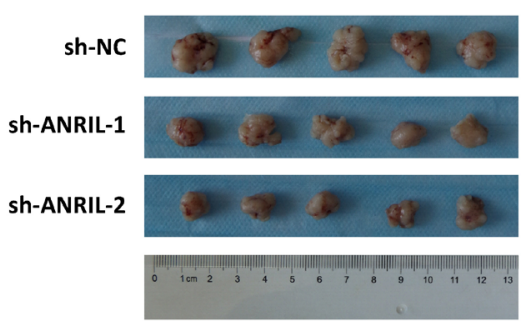

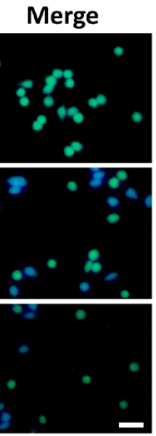

U266

G

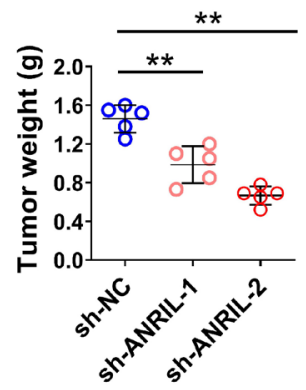

Figure 3. Knockdown of ANRIL suppresses proliferation and tumor xenograft growth of MM cells. A) U266 cells were introduced with sh-ANRIL-1, sh-ANRIL-2, or sh-NC, then the relative ANRIL expression was evaluated by qRT-PCR. B) U266 cells (2,500/well) introduced with sh-ANRIL-1, shANRIL-2, or sh-NC were seeded in 96-well plates, then cell viability was evaluated at day $0,2,4$, and 6 . C-D, U266 cells (5×105/well) introduced with shANRIL-1, sh-ANRIL-2, or sh-NC were seeded in 6-well plates for the BrdU incorporation assay. Represent images (C) and percentage of BrdU positive cells $(D)$ are shown. Scale bar $=50 \mu \mathrm{m}$. E-G, U266 cells $\left(2 \times 10^{6}\right)$ introduced with sh-ANRIL-1, sh-ANRIL-2, or sh-NC were implanted into nude mice for 4 weeks. Tumor growth $(\mathrm{E})$, volume $(\mathrm{F})$, or weight $(\mathrm{G})$ are shown. ${ }^{\star} \mathrm{p} \leq 0.05,{ }^{\star *} \mathrm{p}<0.001$

might be involved in the regulation of gene transcription (Figure 5A). Indeed, ANRIL is demonstrated to regulate gene transcription by interacting with polycomb group proteins such as CBX7, SUZ12, and EZH2 [15, 21]. In the RIP assays, we found that ANRIL was successfully pulled down by EZH2, indicating that ANRIL could bind to EZH2 directly in RPMI-8226 and NCI-H929 cells (Figure 5B). In addition, RPMI-8226 cells transduced with ANRIL expression lentivirus or EV control were subjected to transcriptome RNA sequencing. The 20 most significantly downregulated genes after ANRIL overexpression were depicted in the heatmap (Figure 5C). We were surprised to find that PTEN, a wellcharacterized tumor suppressor, was significantly downregulated by ANRIL. Notably, ANRIL is reported to regulate gene transcription in trans through interacting with EZH2, thus mediates trimethylation of lysine 27 of histone $\mathrm{H} 3$ (H3K27me3) of targeted genes [22, 23]. We speculated that ANRIL might regulate PTEN expression by EZH2-mediated epigenetically silencing. Our results indicated that ANRIL overexpression evidently suppressed PTEN expression and increased downstream phosphorylation of Akt in RPMI-8226 and NCI-H929 cells (Figure 5D). In the ChIP assays, we found that the knockdown of ANRIL reduced the occupancy of EZH2 to the promoter region of PTEN as well as H3K27me3 binding levels (Figure 5E). Besides, two short guide RNAs (sgRNAs) specifically targeting EZH2 (sgEZH2-1 and sgEZH2-2) were constructed and introduced into U266 cells. EZH2 was successfully knocked out by these two sgRNAs (Figure 5F). Furthermore, we found that the levels of PTEN were increased after EZH2 knockout. Taken together, our results indicated that ANRIL epigenetically silenced PTEN by interacting with EZH2.

EZH2 knockout or PTEN restoration abrogates the effects of ANRIL in MM cells. To determine if the effects of ANRIL in MM were associated with EZH2-mediated epigenetically silencing of PTEN, we knocked out EZH2 or restored PTEN expression in RPMI-8226 and NCI-H929 cells, then evaluated for cell viability and IC50 of bortezomib. In cell viability assay, we found that overexpression of ANRIL showed no additional effects on EZH2 knockout or PTEN overexpressed RPMI-8226 and NCI-H929 cells (Figures 6A, $6 \mathrm{~B})$. Furthermore, the IC50 of bortezomib had no significant changes in EZH2 knockout or PTEN overexpressed RPMI-8226 and NCI-H929 cells after ANRIL overexpression (Figures 6C, 6D). These results indicated that EZH2 knockout or PTEN restoration abrogated the effects of ANRIL overexpression on proliferation and bortezomib resistance of MM cells. 


\section{Discussion}

ANRIL has a broad spectrum of cellular functions, including influence on proliferation, senescence, apoptosis, and extracellular matrix remodeling $[15,21]$. Moreover, the lncRNA ANRIL is frequently dysregulated in cancers, such as prostate cancer [24], ovarian cancer [25], hepatocellular carcinoma [26], and colorectal cancer [27]. Furthermore, polymorphisms of ANRIL are correlated with the risk of many cancers, including prostate cancer [28], gastric cancer [29], and glioma [30]. In MM, polymorphism in ANRIL is connected with poor progression-free survival [19]. In addition, upregulation of ANRIL is correlated with advanced ISS stage, decreasing complete response, and shorter progression-free survival of MM patients [31]. Though one study indicates that high ANRIL expression promotes malignant proliferation and cancer stemness properties of MM cells by sponging miR-411-3p and regulating HIF-1a [18], we speculated that this should not cover all the underlying molecular mechanism of ANIRL in MM. In our study, we found that ANRIL promoted proliferation, tumor xenograft growth, and bortezomib tolerance of MM cells via EZH2-mediated epigenetically silencing of PTEN. ANRIL was found to mainly accumulate in the nuclei of MM cells, recruit EZH2
A

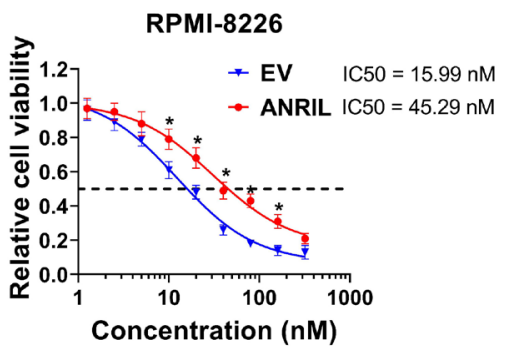

B

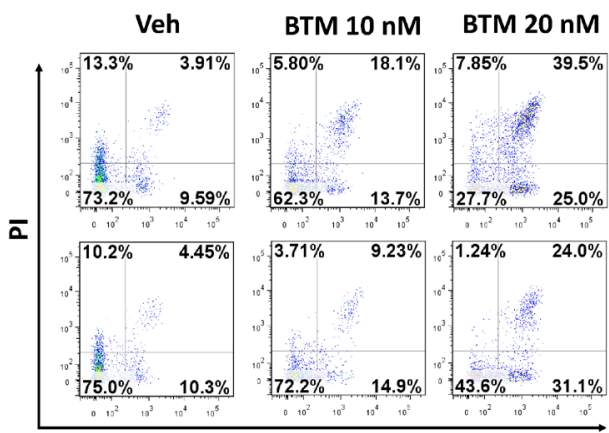

Annexin V

$\mathrm{NCl}-\mathrm{H} 929$

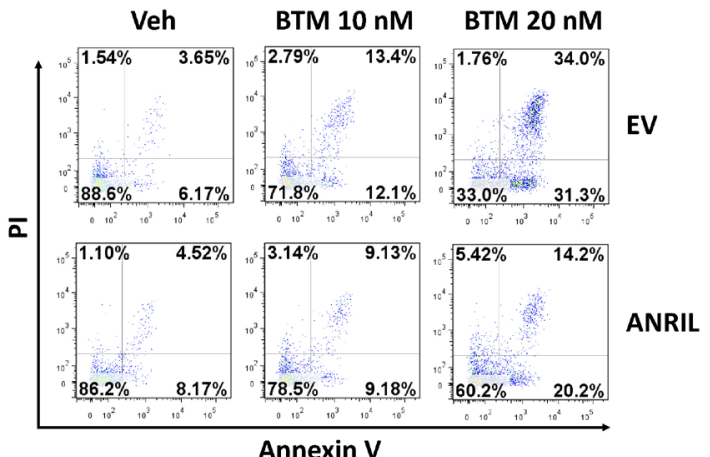

NCl-H929

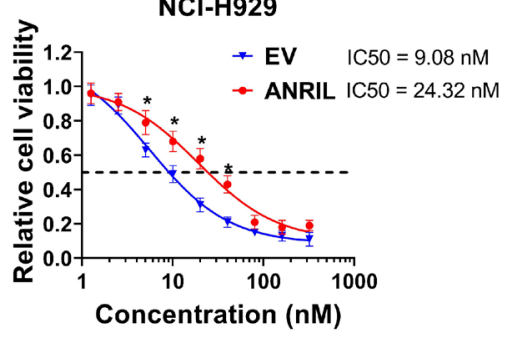

C

D
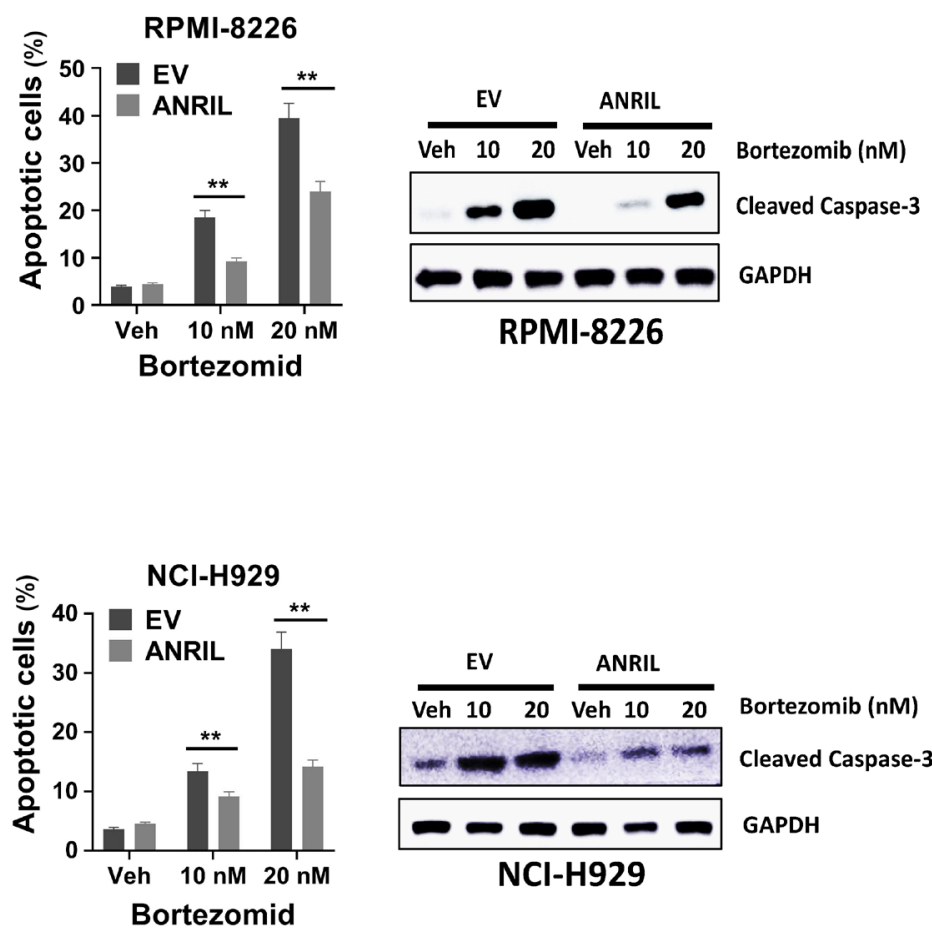

Bortezomib (nM)

Cleaved Caspase-3

GAPDH

Figure 4. ANRIL overexpression increases bortezomib tolerance of MM cells. A) RPMI-8226 and NCI-H929 cells (2,500/well) transduced with ANRIL or EV control were seeded in 96-well plates, then treated with $0,1.25,2.5,5,10,20,40,80,160$, and $320 \mathrm{nM}$ bortezomib for $6 \mathrm{~d}$ and evaluated by cell viability assay. B-D) RPMI-8226 and NCI-H929 cells transduced with ANRIL or EV control were treated with 10 and $20 \mathrm{nM}$ bortezomib or an equal volume of DMSO for $72 \mathrm{~h}$, then cells were stained with Annexin V-FITC and DAPI for flow cytometry (B, C) or protein lysates were collected for western blot (D). Annexin V-FITC and DAPI double-positive apoptotic cell subsets are shown $(\mathrm{C}) .{ }^{*} \mathrm{p} \leq 0.05,{ }^{* *} \mathrm{p}<0.001$ 
A

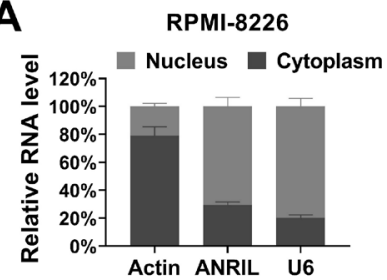

C

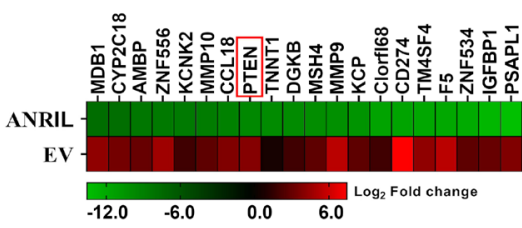

NCI-H929

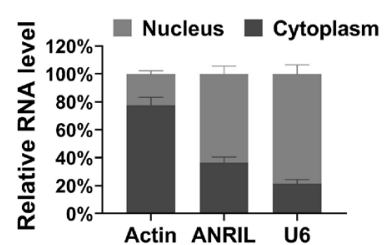

D

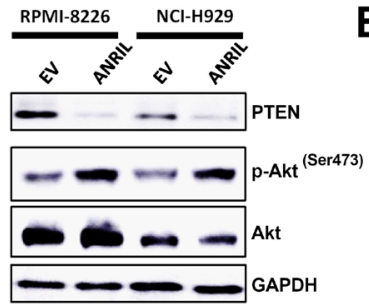

B
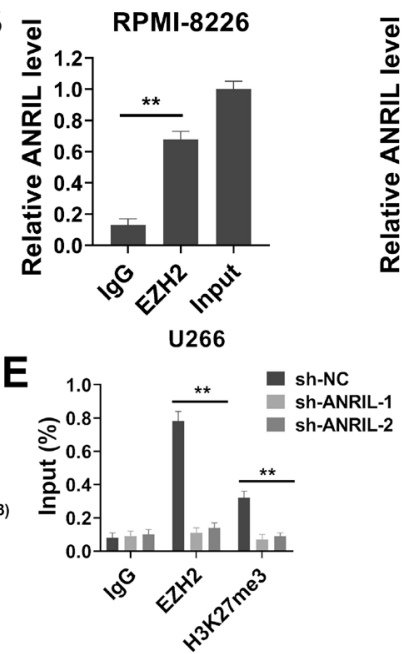
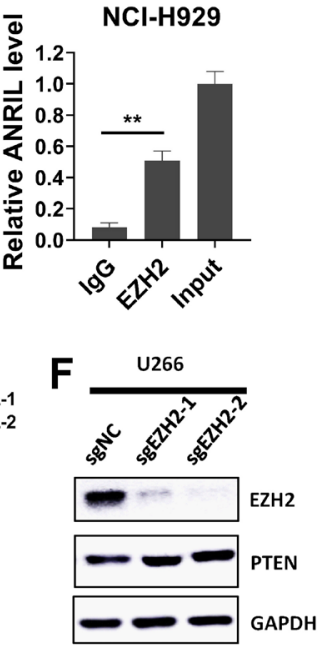

Figure 5. ANRIL epigenetically silences PTEN by interacting with EZH2. A) Relative ANRIL expression in the cytoplasm or the nuclei of RPMI-8226 and NCI-H929 cells were evaluated by qRT-PCR. B) Relative ANRIL levels pulled down by EZH2 or IgG in RIP assay were evaluated by qRT-PCR. C) Heatmap showed the top 20 downregulated genes in transcriptome RNA sequencing. D) Lysates from RPMI-8226 and NCI-H929 cells transduced with ANRIL or EV control were collected for western blot. E) ChIP-PCR analysis of EZH2 occupancy and H3K27me3 binding in the PTEN promoter in U266 cells transduced with sh-ANRIL-1, sh-ANRIL-2, or sh-NC. F) Lysates from U266 cells transduced with sgEZH2-1, sgEZH2-2, or sgNC were collected for western blot. ${ }^{* *} \mathbf{p}<0.001$

A

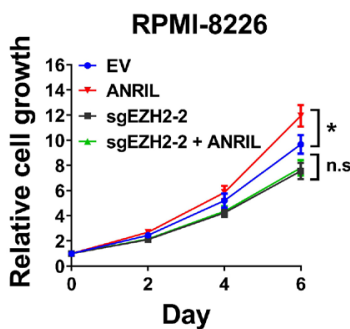

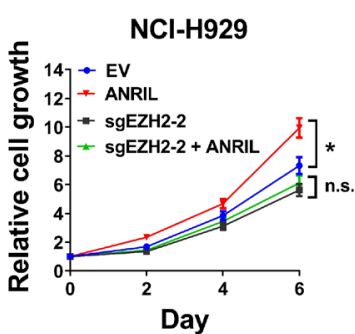

B

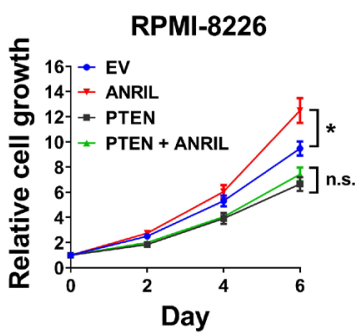

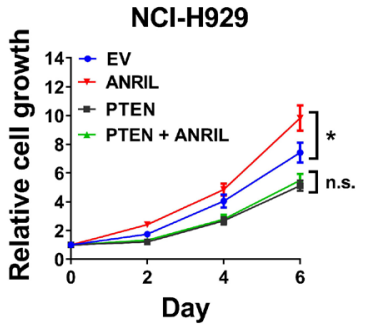

C
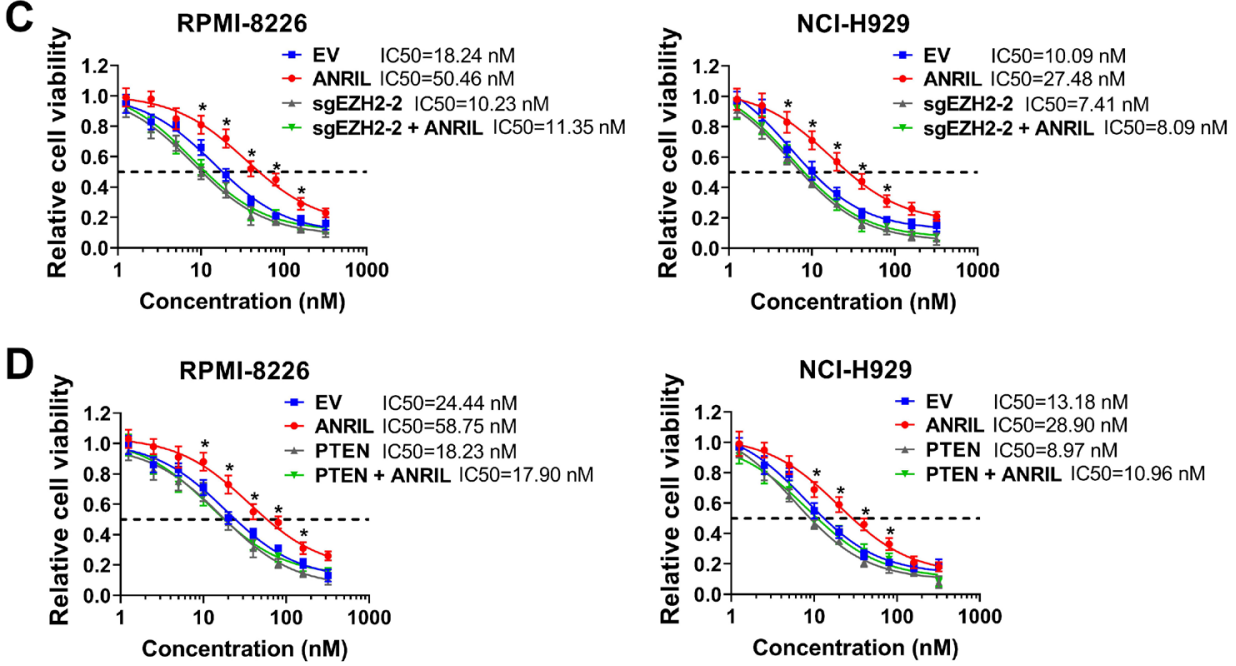

Figure 6. EZH2 knockout or PTEN restoration abrogates the effects of ANRIL in MM cells. A) RPMI-8226 and NCI-H929 cells (2,500/well) transduced with EV, ANRIL, sgEZH2-2, or sgEZH2-2+ANRIL were seeded in 96-well plates, then cell viability was evaluated at day 0, 2, 4, and 6. B) RPMI-8226 and NCI-H929 cells (2,500/well) transduced with EV, ANRIL, PTEN, or PTEN+ANRIL were seeded in 96-well plates, then cell viability was evaluated at day 0, 2, 4, and 6. C) RPMI-8226 and NCI-H929 cells (2,500/well) transduced with EV, ANRIL, sgEZH2-2, or sgEZH2-2+ANRIL were seeded in 96well plates, then treated with $0,1.25,2.5,5,10,20,40,80,160$, and $320 \mathrm{nM}$ bortezomib for $6 \mathrm{~d}$ and evaluated by cell viability assay. D) RPMI-8226 and NCI-H929 cells (2,500/well) transduced with EV, ANRIL, PTEN, or PTEN+ANRIL were seeded in 96-well plates, then treated with 0, 1.25, 2.5, 5, 10, $20,40,80,160$, and $320 \mathrm{nM}$ bortezomib for $6 \mathrm{~d}$ and evaluated by cell viability assay. $n . s$. , not significant. ${ }^{*} \mathrm{p} \leq 0.05$ 
to the promoter region of PTEN, and ultimately increase the H3K27me3 level. Our study provided a novel explanation for the effects of ANRIL overexpression in MM.

ANRIL is reported to be predominately located in the nucleus, especially the linear isoforms of ANRIL that containing the proximal and distal exons [15]. In our study, we demonstrated that ANRIL mainly existed in the nuclear fraction of RPMI-8226 and NCI-H929 cells, indicating that ANRIL resided predominately in the nuclei in MM. Subcellular location is vital for ANRIL to execute its functions. In general, ANRIL can regulate gene transcription in cis and in trans by interacting with polycomb repressive complex (PRC) histone modifiers or sponging microRNAs [15]. In our study, we found that ANRIL interacted with EZH2, a key component of the PRC2 complex. The PRC2 complex can catalyze trimethylation of $\mathrm{H} 3 \mathrm{~K} 27$, thus regulating gene transcription in trans [22]. Indeed, our transcriptome sequencing analysis identified PTEN as a target of ANRIL. ANRIL regulated PTEN expression via EZH2-mediated trimethylation of $\mathrm{H} 3 \mathrm{~K} 27$ at the promoter region of PTEN. PTEN is a well-known tumor suppressor in cancers, and it is also frequently inactivated via different mechanisms. Furthermore, PTEN is an important inhibitor of the PI3K/Akt signaling pathway. In our study, we found that ANRIL overexpression decreased PTEN expression and activated Akt phosphorylation, which might explain the oncogenic functions of ANRIL in MM cells.

Though the survival of MM patients has improved a lot in recent years, most of the MM patients will exhibit resistance to chemotherapy drugs and relapse over time [3]. Bortezomib is the first-line chemotherapy drug used for treating newly diagnosed MM. Intrinsic or acquired resistance to bortezomib frequently occurs during the course of treatment [20]. There is evidence that lncRNAs are also participating in the chemoresistance to bortezomib. For example, upregulation of lncRNA H19 promotes bortezomib resistance of MM cells by sponging miR-29b-3p and increasing MCL-1 expression [32]. LncRNA PRAL is downregulated in primary MM cells and cell lines and correlated with the advanced ISS stage [33]. Moreover, overexpression of PARL suppresses proliferation, induces apoptosis, and enhanced bortezomib sensitivity of MM cells. In our study, overexpression of ANIRL was found to increase bortezomib resistance of MM cells. Bortezomib treatment significantly increased cell apoptosis, but this was diminished by ANRIL overexpression. Our results pointed out the role of ANRIL in the chemoresistance of MM.

In summary, we found that lncRNA ANRIL was upregulated in MM patients and cell lines, and correlated with the advanced ISS stage and poor overall survival. Overexpression of ANRIL promoted proliferation and tumor xenograft growth of MM cells, while knockdown of ANRIL exhibited the contrary effects. In addition, enforced ANRIL expression increased bortezomib tolerance and reduced bortezomibinduced apoptosis in MM cells. ANRIL was mainly distributed in the nucleus and interacted with EZH2, thus increasing the H3K27me3 level of PTEN. Downregulation of PTEN by ANRIL increased the phosphorylation of Akt, while the EZH2 knockout or PTEN restoration abrogated the effects of ANRIL in MM cells. Our results suggested that ANRIL exerted oncogenic functions and conferred chemoresistance of MM cells, thus it might be a novel prognostic marker or therapeutic target for MM.

\section{References}

[1] RAJKUMAR SV, DIMOPOULOS MA, PALUMBO A, BLADE J, MERLINI G et al. International Myeloma Working Group updated criteria for the diagnosis of multiple myeloma. Lancet Oncol 2014; 15: e538-548. https://doi. org/10.1016/S1470-2045(14)70442-5

[2] JEMAL A, SIEGEL R, XU J, WARD E. Cancer statistics, 2010. CA Cancer J Clin 2010; 60: 277-300. https://doi.org/10.3322/ caac. 20073

[3] RAJKUMAR SV, KUMAR S. Multiple Myeloma: Diagnosis and Treatment. Mayo Clin Proc 2016; 91: 101-119. https:// doi.org/10.1016/j.mayocp.2015.11.007

[4] KYLE RA, RAJKUMAR SV. Criteria for diagnosis, staging, risk stratification and response assessment of multiple myeloma. Leukemia 2009; 23: 3-9. https://doi.org/10.1038/ leu.2008.291

[5] KUMAR SK, DISPENZIERI A, LACY MQ, GERTZ MA, BUADI FK et al. Continued improvement in survival in multiple myeloma: changes in early mortality and outcomes in older patients. Leukemia 2014; 28: 1122-1128. https://doi. org/10.1038/leu.2013.313

[6] PAWLYN C, MORGAN GJ. Evolutionary biology of highrisk multiple myeloma. Nat Rev Cancer 2017; 17: 543-556. https://doi.org/10.1038/nrc.2017.63

[7] BHAN A, SOLEIMANI M, MANDAL SS. Long Noncoding RNA and Cancer: A New Paradigm. Cancer Res 2017; 77: 3965-3981. https://doi.org/10.1158/0008-5472.CAN-162634

[8] MAO C, WANG X, LIU Y, WANG M, YAN B et al. A G3BP1Interacting lncRNA Promotes Ferroptosis and Apoptosis in Cancer via Nuclear Sequestration of p53. Cancer Res 2018; 78: 3484-3496. https://doi.org/10.1158/0008-5472.CAN-173454

[9] WANG M, MAO C, OUYANG L, LIU Y, LAI W et al. Long noncoding RNA LINC00336 inhibits ferroptosis in lung cancer by functioning as a competing endogenous RNA. Cell Death Differ 2019; 26: 2329-2343. https://doi.org/10.1038/ s41418-019-0304-y

[10] ZHOU M, ZHAO H, WANG Z, CHENG L, YANG L et al. Identification and validation of potential prognostic lncRNA biomarkers for predicting survival in patients with multiple myeloma. J Exp Clin Cancer Res 2015; 34: 102. https://doi. org/10.1186/s13046-015-0219-5

[11] LU M, HU Y, WU Y, ZHOU H, JIAN Y et al. Genome-wide discovery and characterization of long noncoding RNAs in patients with multiple myeloma. BMC Med Genomics 2019; 12: 135. https://doi.org/10.1186/s12920-019-0577-5 
[12] AMODIO N, STAMATO M A, JULI G, MORELLI E, FULCINITI $M$, et al. Drugging the lncRNA MALAT1 via LNA gapmeR ASO inhibits gene expression of proteasome subunits and triggers anti-multiple myeloma activity. Leukemia 2018; 32: 1948-1957. https://doi.org/10.1038/s41375-0180067-3

[13] RONCHETTI D, TODOERTI K, VINCI C, FAVASULI V, AGNELLI L et al. Expression Pattern and Biological Significance of the lncRNA ST3GAL6-AS1 in Multiple Myeloma. Cancers (Basel) 2020; 12: 782. https://doi.org/10.3390/cancers 12040782

[14] POPOV N, GIL J. Epigenetic regulation of the INK4b-ARFINK4a locus: in sickness and in health. Epigenetics 2010; 5: 685-690. https://doi.org/10.4161/epi.5.8.12996

[15] KONG Y, HSIEH CH, ALONSO LC. ANRIL: A lncRNA at the CDKN2A/B Locus With Roles in Cancer and Metabolic Disease. Front Endocrinol (Lausanne) 2018; 9: 405. https:// doi.org/10.3389/fendo.2018.00405

[16] SUN LY, LI XJ, SUN YM, HUANG W, FANG K et al. LncRNA ANRIL regulates AML development through modulating the glucose metabolism pathway of AdipoR1/AMPK/ SIRT1. Mol Cancer 2018; 17: 127. https://doi.org/10.1186/ s12943-018-0879-9

[17] LI G, GAO L, ZHAO J, LIU D, LI H et al. LncRNA ANRIL/ miR-7-5p/TCF4 axis contributes to the progression of T cell acute lymphoblastic leukemia. Cancer Cell Int 2020; 20: 335. https://doi.org/10.1186/s12935-020-01376-8

[18] WANG M, ZHAO HY, ZHANG JL, WAN DM, LI YM et al. Dysregulation of LncRNA ANRIL mediated by miR-411$3 p$ inhibits the malignant proliferation and tumor stem cell like property of multiple myeloma via hypoxia-inducible factor 1alpha. Exp Cell Res 2020; 396: 112280. https://doi. org/10.1016/j.yexcr.2020.112280

[19] POI MJ, LI J, SBOROV DW, VANGUNDY Z, CHO YK et al. Polymorphism in ANRIL is associated with relapse in patients with multiple myeloma after autologous stem cell transplant. Mol Carcinog 2017; 56: 1722-1732. https://doi. org/10.1002/mc.22626

[20] MURRAY MY, AUGER MJ, BOWLES KM. Overcoming bortezomib resistance in multiple myeloma. Biochem Soc Trans 2014; 42: 804-808. https://doi.org/10.1042/ BST20140126

[21] AGUILO F, DI CECILIA S, WALSH MJ. Long Non-coding RNA ANRIL and Polycomb in Human Cancers and Cardiovascular Disease. Curr Top Microbiol Immunol 2016; 394 : 29-39. https://doi.org/10.1007/82_2015_455

[22] MARGUERON R, LI G, SARMA K, BLAIS A, ZAVADIL J et al. Ezh1 and Ezh2 maintain repressive chromatin through different mechanisms. Mol Cell 2008; 32: 503-518. https:// doi.org/10.1016/j.molcel.2008.11.004

[23] YU Y, CHEN Q, ZHANG X, YANG J, LIN K et al. Long noncoding RNA ANRIL promotes the malignant progression of cholangiocarcinoma by epigenetically repressing ERRFI1 expression. Cancer Sci 2020; 111: 2297-2309. https://doi. org/10.1111/cas. 14447
[24] ZHAO B, LU YL, YANG Y, HU LB, BAI Y et al. Overexpression of lncRNA ANRIL promoted the proliferation and migration of prostate cancer cells via regulating let-7a/TGFbeta1/ Smad signaling pathway. Cancer Biomark 2018; 21: 613-620. https://doi.org/10.3233/CBM-170683

[25] MIAO JT, GAO JH, CHEN YQ, CHEN H, MENG HY et al. LncRNA ANRIL affects the sensitivity of ovarian cancer to cisplatin via regulation of let-7a/HMGA2 axis. Biosci Rep 2019; 39: BSR20182101. https://doi.org/10.1042/ BSR20182101

[26] MA Y, ZHANG H, LI G, HU J, LIU X et al. LncRNA ANRIL promotes cell growth, migration and invasion of hepatocellular carcinoma cells via sponging miR-144. Anticancer Drugs 2019; 30: 1013-1021. https://doi.org/10.1097/ CAD.0000000000000807

[27] NAEMURA M, TSUNODA T, INOUE Y, OKAMOTO H, SHIRASAWA $S$ et al. ANRIL regulates the proliferation of human colorectal cancer cells in both two- and three-dimensional culture. Mol Cell Biochem 2016; 412: 141-146. https:// doi.org/10.1007/s11010-015-2618-5

[28] TAHERI M, POURESMAEILI F, OMRANI MD, HABIBI M, SARRAFZADEH $S$ et al. Association of ANRIL gene polymorphisms with prostate cancer and benign prostatic hyperplasia in an Iranian population. Biomark Med 2017; 11: 413-422. https://doi.org/10.2217/bmm-2016-0378

[29] PETKEVICIUS V, STRELECKIENE G, BALCIUTE K, LINK A, LEJA $M$ et al. Association of Long Non-Coding RNA Polymorphisms with Gastric Cancer and Atrophic Gastritis. Genes (Basel) 2020; 11: 1505. https://doi.org/10.3390/ genes11121505

[30] DENG Y, ZHOU L, LI N, WANG M, YAO L et al. Impact of four lncRNA polymorphisms (rs2151280, rs7763881, rs1136410, and rs3787016) on glioma risk and prognosis: A case-control study. Mol Carcinog 2019; 58: 2218-2229. https://doi.org/10.1002/mc.23110

[31] YIN Y, YANG W, ZHANG L, LIU K, LUO Z. Long non-coding RNA ANRIL and its target microRNAs (microRNA-34a, microRNA-125a and microRNA-186) relate to risk stratification and prognosis in multiple myeloma. Hematology 2021; 26: 160-169. https://doi.org/10.1080/16078454.2021.187227 5

[32] PAN Y, ZHANG Y, LIU W, HUANG Y, SHEN X et al. LncRNA H19 overexpression induces bortezomib resistance in multiple myeloma by targeting MCL-1 via miR-29b-3p. Cell Death Dis 2019; 10: 106. https://doi.org/10.1038/s41419018-1219-0

[33] XIAO G, LI Y, WANG Y, ZHAO B, ZOU Z et al. LncRNA PRAL is closely related to clinical prognosis of multiple myeloma and the bortezomib sensitivity. Exp Cell Res 2018; 370: 254-263. https://doi.org/10.1016/j.yexcr.2018.06.026 